\title{
AUTO-SINTONIA CONSERVATIVA PARA CONTROLE PREDITIVO GENERALIZADO ADAPTATIVO
}

\author{
Daniel Cavalcanti Jeronymo*, Rejane De Barros Araujo*, Antonio Augusto Rodrigues \\ COELHO* \\ * Universidade Federal de Santa Catarina \\ Pós-Graduação em Engenharia de Automação e Sistemas \\ Campus Universitário Reitor João David Ferreira Lima, C.P. 476, CEP 88040-900 \\ Florianópolis, SC, Brasil
}

Emails: dcavalcanti@bigfoot.com, rejane@das.ufsc.br, aarc@das.ufsc.br

\begin{abstract}
This paper presents a method for conservative self-tuning for adaptive Generalized Predictive Control (GPC), using recursive least squares for model estimation. The process estimation error is utilized to create a trust factor which adjusts GPC parameters in order to generate a more conservative control action when facing process uncertainties. In order to validate the proposed method, two study cases were used, the first a simulation of a non-linear control valve and the second a real application on a non-linear underdamped pendulum. The proposed GPC self-tuning method has its performance compared to the classic GPC, demonstrating superior results regarding the smoothness of the control action especially regarding the treatment of ringing.
\end{abstract}

Keywords - generalized predictive control, non linear control, ringing, self-tuning.

Resumo- Neste trabalho é apresentado um método de auto-sintonia ( self-tuning) com características conservativas para o GPC adaptativo, utilizando mínimos quadrados recursivo para estimação do modelo. O erro de estimação do processo é utilizado para criar um fator de confiança que ajusta os parâmetros do GPC para tornar a ação de controle mais conservativa frente a incertezas de processo. Para validação deste método foram utilizados dois estudos de caso, o primeiro uma simulação duma válvula de controle não-linear e o segundo uma aplicação real num pendulo não-linear sub-amortecido. O método de auto-sintonia proposto é comparado com o GPC clássico, demonstrando resultados superiores quanto a suavidade da ação de controle especialmente quanto ao tratamento de ringing.

Palavras-chave — controle preditivo generalizado, controle não linear, ringing, auto-sintonia.

\section{Introdução}

O controle preditivo generalizado (Clarke et al., 1987a; Clarke et al., 1987b) é fundamentado no uso de predições da resposta do sistema e das ações de controle, buscando minimizar uma função custo que consiste no somatório do erro quadrático entre a resposta estimada do sistema e a referência e também um somatório das ações de controle futuro. Este controlador é aplicável a sistemas de fase não-mínima, instáveis em malha aberta, com tempo-morto ou ordem desconhecida (Pivoňka and Nepevný, 2005). Já foi demonstrado que o GPC adaptativo pode atingir melhores resultados que o GPC clássico quanto a robustez frente a perturbações e melhores características de rastreamento de referência (Zidane et al., 2011; Škrjanc, 2007).

Neste trabalho é apresentado um novo método para auto-sintonia do GPC adaptativo utilizando o erro do estimador como um fator de confiança para a sintonia dos parâmetros do GPC. Com o fator de confiança o GPC adaptativo pode assumir uma postura mais agressiva quando há certeza do modelo estimado e ações mais conservativas quando há incertezas na estimação. Desta maneira, o controlador pode trafegar entre zonas de operação sem perda de desempenho.

Para validar o método proposto, este foi implementado para uma simulação de uma válvula de controle não-linear de modelo Wiener e para uma aplicação real de um pendulo amortecido. Os resultados obtidos demonstram a eficácia do uso do fator de confiança na sintonia dos parâmetros do GPC.

O restante deste trabalho está organizado da seguinte maneira. A seção 2 faz uma breve introdução ao GPC e seus parâmetros. A seção 3 apresenta os fundamentos do método de autosintonia proposto. A seção 4 apresenta os casos de estudo, uma planta não-linear de válvula de controle simulada e uma aplicação real de um pendulo não-linear subamortecido. A seção 5 apresenta os resultados obtidos pelo método proposto e os compara com o GPC clássico. Finalmente, a seção 6 apresenta as considerações finais sobre o trabalho e trabalhos futuros.

\section{Controle Preditivo Generalizado Adaptativo}

O controle preditivo generalizado (Clarke et al., 1987a; Clarke et al., 1987b) é um dos controladores baseados em modelo mais comuns sendo amplamente utilizado tanto na academia quanto na indústria. Neste tipo de controlador as ações de controle são calculadas buscando minimizar uma função custo, o erro quadrático entre a referência e as saídas futuras da planta. Estas saídas futuras são estimadas por um modelo linear do 
processo, geralmente obtido por identificação do sistema. A ação de controle do GPC é caracterizada por $\Delta u=\left(G^{\prime} G+\lambda\right)^{-1} G^{\prime}\left(y_{r}-f\right)$, onde $G$ é uma matriz de parâmetros da resposta ao degrau do sistema, $y_{r}$ é a referência para rastreamento de referência e $f$ a resposta livre que depende de ações de controle passadas. Como parâmetros o GPC utiliza o horizonte de predição $N_{y}$, o horizonte de controle $N_{u}$ e o fator de ponderação de controle $\lambda$.

Dada a necessidade de modelo, em aplicações de controle adaptativo o GPC é utilizado em conjunto com um estimador. Neste trabalho foi utilizado o mínimos quadrados recursivo (Recursive Least Squares - LSR). Neste estimador é considerado o erro quadrático entre os valores observados do processo e os valores estimados, sendo possível assim a obtenção de um modelo ARX (autoregressive exogenous). Tem como parâmetros na, o número de pólos do modelo, nb, o número de zeros e $P_{0}$, o valor de inicialização da matriz de covariância do estimador.

\section{Auto-sintonia conservativa}

Na maioria das aplicações de controle os parâmetros de sintonia do controlador são fixos ao longo da operação do sistema, o que pode acarretar em degeneração do comportamento esperado em sistemas não-lineares ao longo de múltiplas zonas de operação. Para contornar este problema, uma solução clássica é o gain scheduling (Leith and Leithead, 2000). Entretanto, em aplicações de controle adaptativo o gain scheduling não pode ser utilizado já que não se conhece o comportamento do sistema para sintonizar o controlador $a$ priori.

Neste trabalho propomos a utilização de um fator de confiança $K_{T}$ fundamentado no erro do estimador $e(k)$ para ajustar os parâmetros do controlador:

$$
K_{T}=1-\frac{e(k)}{e(k)_{\max }},
$$

onde $e(k)_{\max }$ é o maior erro de estimação encontrado durante o controle. O fator de confiança permite utilizar as incertezas do estimador como segurança na auto-sintonia dos parâmetros utilizados, quando $K_{T}$ tende a 1, o modelo identificado do processo representa adequadamente.

\subsection{Horizonte de predição}

Para a sintonia do horizonte de predição $N_{y}$ é determinado a priori um valor máximo $N_{y \max }$, em seguida é utilizado o valor real do pólo mais lento $\tau_{\text {slow }}$ do modelo $B\left(z^{-1}\right) / A\left(z^{-1}\right)$ obtido pelo estimador.

Considerando um tempo de acomodação de $95 \%$, tem-se:

$$
N_{y}=\operatorname{round}\left(K_{T} 3 \frac{\tau_{\text {slow }}}{T_{s}}\right),
$$

onde o horizonte de predição é então saturado entre $1 \leq N_{y} \leq N_{\text {ymax }}$.

\subsection{Horizonte de controle}

Para a sintonia do horizonte de controle é utilizada a quantidade de pólos instáveis $N_{u p}$ do modelo $B\left(z^{-1}\right) / A\left(z^{-1}\right)$ obtido pelo estimador, considerando a sugestão da literatura (Clarke et al., 1987a) $N_{u}$ deve ser grande o suficiente para conter pólos instáveis ou oscilatórios (mal-amortecidos), portanto.:

$$
N_{u}=1+N_{u p}
$$

\subsection{Fator de ponderação de controle}

Para a sintonia do fator de ponderação de controle devem ser determinados a priori dois valores. Primeiro, um valor mínimo $\lambda_{\min }$ que a ponderação de controle deve assumir considerando certeza absoluta do processo. Segundo, um valor $\lambda_{\max }$ que na ausência absoluta de certeza do processo resulte numa ponderação satisfatória. Com estes valores a auto-sintonia dá-se por:

$$
\lambda=\lambda_{\max }\left(1-K_{T}\right)+\lambda_{\min } .
$$

Embora a existência de dois parâmetros para a sintonia do fator de ponderação de controle aumente a complexidade de projeto, ganha-se a flexibilidade de maior ponderação de controle frente a erros não esperados de modelagem.

\subsection{Sintese do método de auto-sintonia}

Inicialmente devem ser definidos a priori $N_{y \max }$, $\lambda_{\min }, \lambda_{\max }$ e a cada instante de controle devem ser calculados:

$$
\begin{aligned}
& \text { 1. } e(k)_{\max } \\
& \text { 2. } \tau_{\text {slow }} \\
& \text { 3. } N_{\text {up }} \\
& \text { 4. } K_{T}=1-\frac{e(k)}{e(k)_{\max }} \\
& \text { 5. } N_{y}=\operatorname{round}\left(K_{T} 3 \frac{\tau_{\text {slow }}}{T_{s}}\right) \\
& \text { 6. } 1 \leq N_{y} \leq N_{\text {ymax }} \\
& \text { 7. } \lambda=\lambda_{\max }\left(1-K_{T}\right)+\lambda_{\text {min }}
\end{aligned}
$$

\section{Casos de estudo}

Para avaliar o método apresentado foram utilizados dois sistemas não-lineares. O primeiro, a simulação de uma válvula de controle. O segundo, uma aplicação real de um pendulo subamortecido. 


\subsection{Simulação - Válvula de Controle}

O sistema não-linear da válvula de controle pode ser encontrado na literatura (Wigren, 1990; AlDuwaish and Naeem, 2001). O sistema é depictado na figura 1, adaptada de Cable (2005).

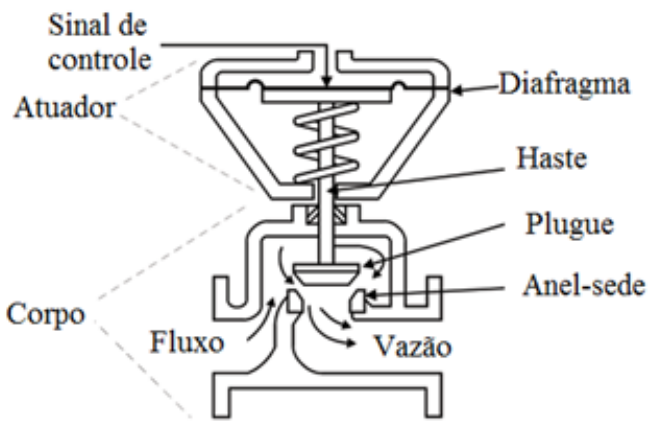

Figura 1: Diagrama do sistema válvula de controle.

Este processo é descrito pelo modelo Wiener:

$$
\begin{aligned}
& x(t)=\frac{0,616 z^{-1}+0,05343 z^{-2}}{1-1,5714 z^{-1}+0,6873 z^{-2}} u(t), \\
& y(t)=\frac{x(t)}{\sqrt{0,1+0,9 x^{2}(t)}} .
\end{aligned}
$$

O processo tem como limitação a entrada de controle $u(k)$ no domínio [00,4] e tempo de amostragem $T_{s}=0,1$. A curva estática é apresentada na figura 2 onde é possível observar um claro comportamento não-linear na saída do processo.

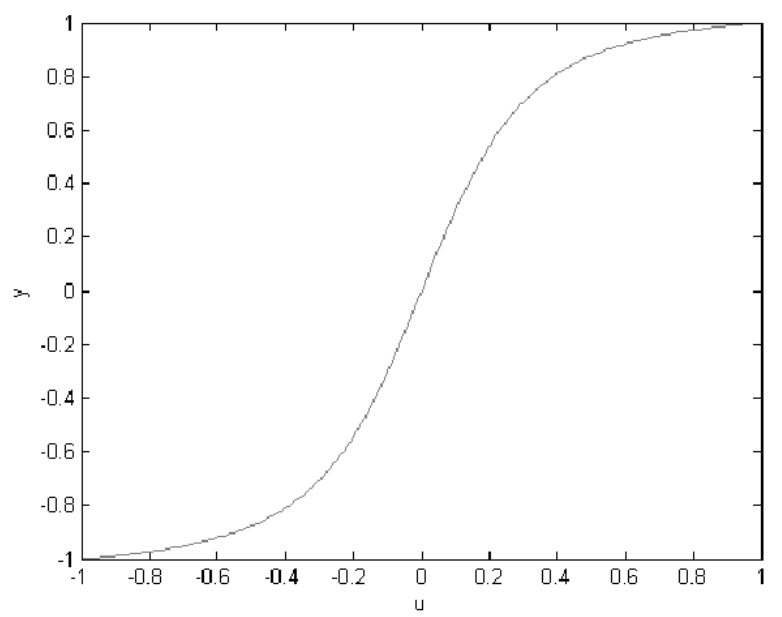

Figura 2: Característica de entrada-saída do sistema válvula de controle.

\subsection{Aplicação Real - Pendulo subamortecido}

O pendulo não-linear subamortecido, também conhecido como pendulo amortecido, é apresentado na figura 3. Este processo contém uma barra vertical onde há um potenciômetro no eixo central para medir a posição angular $\theta$. No extremo superior da barra há uma massa e no inferior um sistema de propulsão constituído de um motor DC e uma hélice. Quando uma tensão de entrada $u$ é aplicada a posição angular da barra é modificada. O objetivo é posicionar a barra em um angulo de referência.

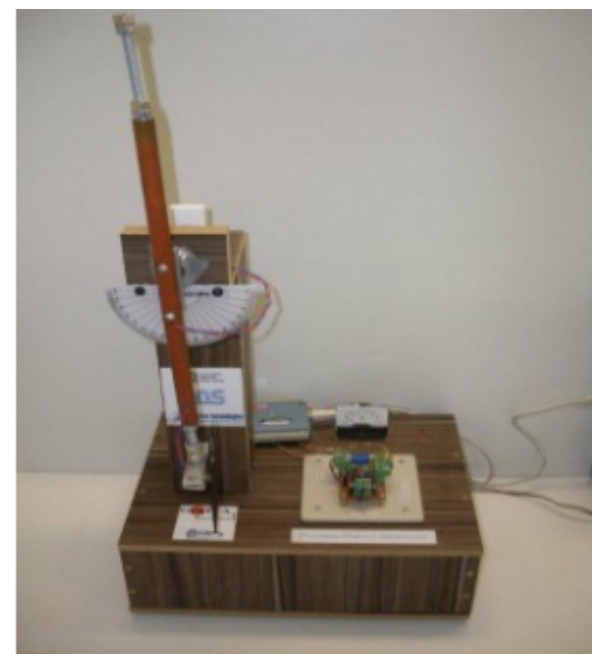

Figura 3: Processo pendulo amortecido.

O processo tem como limitação a entrada de controle $u(k)$ no domínio [04,9] e tempo de amostragem $T_{s}=0,1$. A curva estática é apresentada na figura 4 , onde se observa duas zonas mortas e entre elas um comportamento levemente nãolinear.

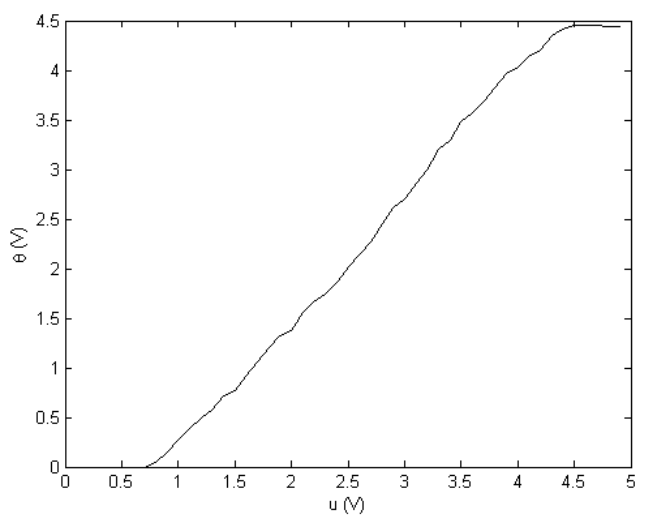

Figura 4: Característica de entrada-saída do sistema pendulo amortecido.

\section{Resultados}

No processo válvula de controle, para o estimador foram utilizados os parâmetros $n a=2, n b=2$ e $P_{0}=1000$, para o GPC clássico $N_{y}=20$, $N_{u}=5, \lambda=7,9$ e para o GPC com auto-sintonia 
$N_{y \max }=20, \lambda_{\max }=5$ e $\lambda_{\min }=7,9$. O processo foi simulado para quase 500 segundos com cinco trocas de referência. Os resultados para o GPC clássico podem ser observados na figura 5 e para o GPC com auto-sintonia na figura 6. Observa-se para o GPC com auto-sintonia por estes resultados a diminuição do ringing nas trocas de referência enquanto o processo mantem aproximadamente o mesmo tempo de acomodação, porém com um comportamento muito mais conservativo.
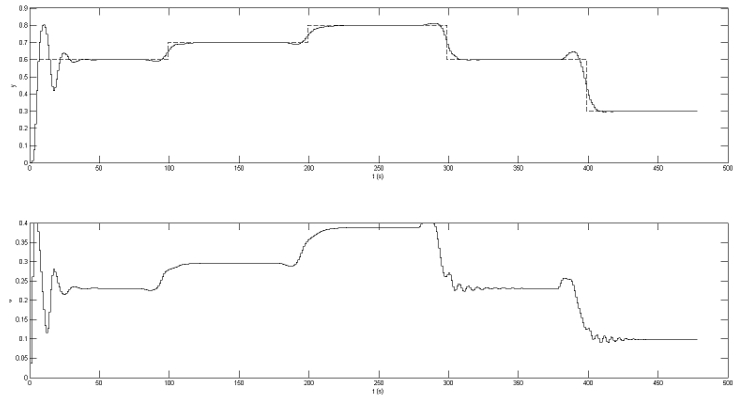

Figura 5: Resultado do GPC adaptativo clássico para a válvula de controle.
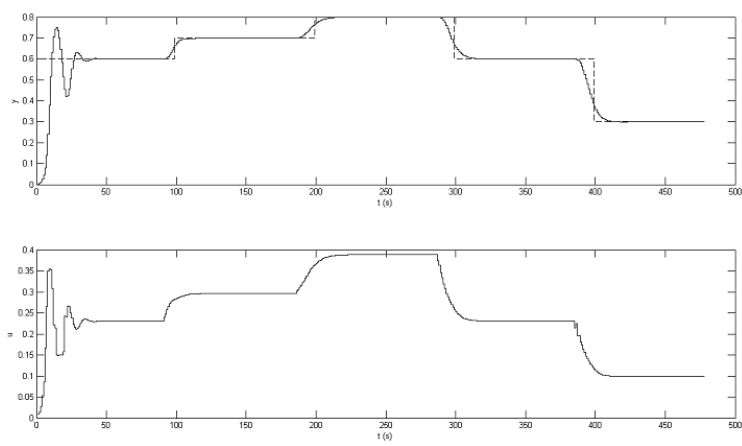

Figura 6: Resultado do GPC adaptativo com auto-sintonia para a válvula de controle.

No processo válvula de controle, para o estimador foram utilizados os parâmetros $n a=2$, $n b=2$ e $P_{0}=100$, para o GPC clássico $N_{y}=10$, $N_{u}=1, \lambda=20$ e para o GPC com auto-sintonia $N_{y \max }=10, \lambda_{\max }=150$ e $\lambda_{\min }=50$. O processo foi simulado para quase 100 segundos com duas trocas de referência. Os resultados para o GPC clássico podem ser observados na figura 7 e para o GPC com auto-sintonia na figura 8. Não se observa diferenças significativas quanto a agressividade ou ringing na ação de controle para estes resultados, porém o GPC clássico demonstrou uma incapacidade em lidar com a diferença entre as zonas de operação enquanto o GPC com auto-sintonia operou de forma mais eficaz, atingindo um menor tempo de acomodação para este cenário.

Observa-se para estes resultados um melhor desempenho do GPC com auto-sintonia em rela-
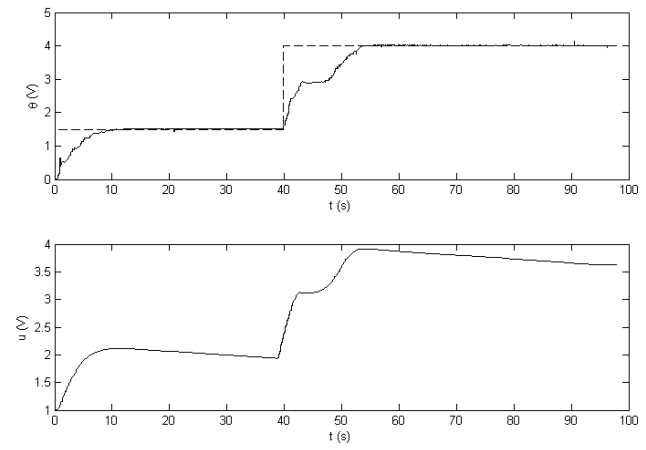

Figura 7: Resultado do GPC adaptativo clássico para o pendulo amortecido.
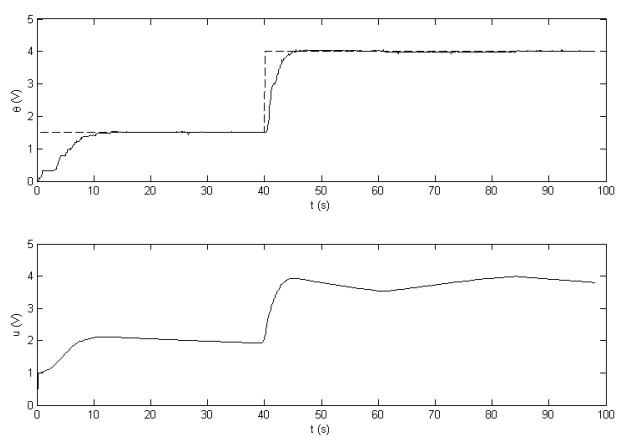

Figura 8: Resultado do GPC adaptativo com auto-sintonia para o pendulo amortecido. 
ção ao GPC clássico quanto as mudanças entre zonas de operação dos sistemas.

\section{Conclusões}

Neste trabalho foi apresentado um método de auto-sintonia para o GPC adaptativo utilizando um fator de confiança fundamentado no erro do estimador. O fator de confiança é utilizado para determinar os horizontes de predição e controle, assim como o fator de ponderação de controle. Quando o fator de confiança é alto, tendendo a 1 , tem-se uma certeza de que o estimador do controlador adaptativo está adequadamente representando o processo controlado e com isso pode-se atingir um desempenho mais agressivo. Pelo outro lado, quando o fator de confiança é baixo, próximo a 0 , tem-se uma certeza de que o estimador não está representando adequadamente o processo controlado e deve-se tomar ações de controle conservativas. Assim, quando o fator de confiança é alto toma-se um horizonte de predição maior e uma menor ponderação de controle. De maneira inversamente equivalente, quando o fator de confiança é baixo toma-se um horizonte de predição menor e uma maior ponderação de controle. $\mathrm{O}$ horizonte de controle é calculado pela quantidade de pólos instáveis do modelo estimado.

O método proposto foi implementado no MATLAB e comparado com o GPC adaptativo clássico, isto é, com parâmetros sintonizados a priori e invariantes. Para avaliação do método, este foi aplicado a uma simulação de uma válvula de controle não-linear descrita por um processo Wiener, assim como a uma aplicação real de um pendulo amortecido.

Resultados obtidos demonstram que o método foi capaz de reduzir o ringing observado pelo GPC clássico para a válvula de controle sem diminuir o tempo de acomodação. Para o pendulo amortecido não foram observadas mudanças significativas quanto a ação de controle porém observou-se uma diminuição no tempo de acomodação numa troca abrupta entre zonas de operação.

Trabalhos futuros irão se concentrar em utilizar o fator de confiança para sintonizar o horizonte de controle, maneiras alternativas para a sintonia do fator de ponderação de controle e também tornar o método independente de conhecimento $a$ priori na forma de parâmetros.

\section{Agradecimentos}

O primeiro autor agradece a UFSC pela concessão de uma bolsa de doutorado vinculada ao $\mathrm{CNPq}$ (processo: 140592/2012-1) e a segunda autora pela concessão de uma bolsa de doutorado vinculada a CAPES.

\section{Referências}

Al-Duwaish, H. and Naeem, W. (2001). Nonlinear model predictive control of hammerstein and wiener models using genetic algorithms, Control Applications, 2001. (CCA '01). Proceedings of the 2001 IEEE International Conference on, pp. 465-469.

Cable, M. (2005). Calibration: A Technician's Guide, ISA Press, Research Triangle Park, USA.

Clarke, D. W., Mohtadi, C. and Tuffs, P. S. (1987a). Generalized predictive control Part I. The basic algorithm, Automatica 23(2): $\quad 137-148$. 10.1016/0005-1098(87)90088-4

Clarke, D. W., Mohtadi, C. and Tuffs, P. S. (1987b). Generalized predictive control - part ii. extensions and interpretations, Automatica 23(2): 149-160. DOI: 10.1016/0005-1098(87)90087-2

Leith, D. J. and Leithead, W. E. (2000). Survey of gain-scheduling analysis and design, International Journal of Control 73(11): 1001-1025. DOI: $10.1080 / 002071700411304$

Pivoňka, P. and Nepevný, P. (2005). Generalized predictive control with adaptive model based on neural networks, Proceedings of the 6th WSEAS international conference on Neural networks, NN'05, pp. 1-4.

Škrjanc, I. (2007). Self-adaptive generalized predictive control of batch reactor, Proceedings of the 26th IASTED International Conference on Modelling, Identification, and Control, MIC '07, ACTA Press, Anaheim, CA, USA, pp. 291-295.

Wigren, T. (1990). Recursive identification based on the nonlinear Wiener model, $\mathrm{PhD}$ thesis, Uppsala University, Disciplinary Domain of Science and Technology.

Zidane, Z., Lafkih, M. and Ramzi, M. (2011). Adaptive generalized predictive control of a heat exchanger pilot plant, Multimedia Computing and Systems (ICMCS), 2011 International Conference on, pp. 1-6. 